\title{
Ciudadanía, comunicación y jóvenes: estado del arte
}

Citizenship, communication and youth: state of art

María Cristina Rodríguez García*

Universidad Anáhuac México

Av. Universidad Anáhuac núm. 46, Col. Lomas Anáhuac,

C.P. 52786, Huixquilucan, Estado de México

Editor: Rogelio del Prado Flores

Fecha de recepción: 6 de abril de 2020

Fecha de aceptación: 9 de mayo de 2020 macrisroga@yahoo.com.mx

https://orcid.org/0000-0002-5740-2356

https://doi.org/10.36105/stx.2020n5.09

\section{RESUMEN}

Desde el 2008 se identifica un aumento en la producción de artículos sobre ciudadanía y jóvenes, con el tiempo los estudios han ido incorporando además la categoría de la comunicación como factor relevante para comprender las nuevas formas ciudadanas de los jóvenes. El propósito de este estudio es aportar un panorama general de los estudios sobre ciudadanía, jóvenes y comunicación para aproximarse a un estado del arte en el estudio de estas tres categorías. El análisis permitirá explorar tendencias metodológicas y de abordaje de los objetos de estudio. Se revisaron 36 artículos científicos publicados en revistas indexadas del periodo 201 I al 2018 , que se desprenden del estado del arte de la tesis doctoral: “Ciudadana y comunicación: configuración de la participación ciudadana, compromiso cívico y orientaciones ciudadanas a partir de la interacción y las mediaciones individuales y culturales en jóvenes mexicanos”. Los resultados mostraron que metodológicamente los países latinoamericanos se orientan a los enfoques cualitativos, además hay una ligera tendencia a los estudios mixtos en los últimos años y enfocados a contextos comunicativos más específicos. En cuanto al análisis de los temas abordados, se observó que los estudios comienzan siendo generales y abordan de manera difusa los aspectos comunicativos, pero con los años los estudios van siendo más claros y determinados desde el enfoque comunicativo.

Palabras clave: ciudadanía, comunicación, jóvenes, estado del arte.

* Doctorante en Investigación de la Comunicación por la Universidad Anáhuac México; Maestra y Licenciada en Psicología, orientada hacia la formación en incidencia ciudadana, participación y discursos de causas sociales. 


\begin{abstract}
Since 2008 , the production of studies on citizenship and youth has increase, incorporating the category of communication as a relevant aspect to understand the citizenship in young people. The purpose of this study is to provide a general overview of the studies regarding citizenship, youth, and communication to show a state of the art in the study of these three categories. The methodology consisted in the analysis of 36 scientific articles published in indexed journals between 20II and 2018. These articles allowed the exploration of tendencies regarding methodology and theoretical approaches to the object of study, the articles were taken form the state of art from a doctoral phd thesis in progress. The results demonstrated that Latin American countries are oriented towards qualitative methodology, additionally, in the last years, it was observed a slight increase in the tendency towards mixed studies and a focus in specific communicational contexts. Regarding the analysis of the topics covered, it was observed the studies, in the beginning, are more general and approached ambiguously the communicative aspects however in the course of the years the become more clear and specific from the communicative perspective.
\end{abstract}

Keywords: Citizenship, Communication, Youth, State of art.

\title{
INTRODUCCIÓN
}

$\mathrm{E}$ labordaje de la ciudadanía, como un estatus que se establece en una relación de pertenencia a un estado que genera deberes y derechos recíprocos (Lizcano, 20I2), desde la teoría política ha sido ampliamente estudiado desde la segunda mitad del siglo pasado con el auge de las democracias en el mundo occidental. El interés por el estudio de la ciudadanía se ha incrementado aún más a partir de la presencia de fenómenos comportamentales como la baja participación política formal, es especial de poblaciones jóvenes, la baja confianza en las instituciones democráticas y el rechazo a la política (Meyer, 200I).

A partir del nuevo milenio, la necesidad de estudiar la ciudadanía en la población de los jóvenes se ha vuelto relevante, por ser el grupo poblacional con mayores cambios en comportamientos y prácticas de la ciudadanía (Padilla de la Torre, 20I4). Livingston (2008) señala que los estudios sobre ciudadanía y participación comenzaron a incrementar a partir del 2008, ante la necesidad de comprender la relación de los jóvenes con nuevas formas de participación y la presencia de cambios comportamentales en cuanto a la participación formal.

El objetivo del presente trabajo es analizar las investigaciones sobre el tema de ciudadanía, jóvenes y comunicación. Se seleccionaron 36 artículos relevantes y representativos, del 
periodo 20 Ir al 20I8, que abordan la ciudadanía en los jóvenes como categoría central y que buscan integrar elementos de la comunicación.

Se presenta primero, un análisis general a modo de línea del tiempo con especial énfasis en el análisis de la tendencia metodológica. Posteriormente, se analiza por años los temas de estudio abordados en los artículos, para detectar algunos cambios y tendencias en la incorporación de la comunicación a los estudios de ciudadanía en jóvenes.

El propósito de la investigación es presentar el análisis de las tendencias en investigación y los cambios que se han presentado en el periodo de tiempo seleccionado, que permita presentar un panorama general del estado del arte en los estudios que abordan la comunicación, la ciudadanía y los jóvenes.

\section{METODOLOGÍA}

Se realizó un análisis descriptivo de los artículos seleccionados de acuerdo al periodo de tiempo establecido, 20II-20I8. Se analizaron 36 artículos en el periodo que cubrieran los requisitos de abordar los tres ejes: ciudadanía, jóvenes y comunicación, que presentaran con claridad la metodología estudiada y que estuviera en revistas indexadas. Los estudios fueron seleccionados con base en que cumplieran los siguientes requisitos: que contemplaran las variables de jóvenes, ciudadanía y comunicación (aunque no fueran del área, pero si contuvieran al menos variables comunicativas); que fueran publicados en revistas indexadas; que fueran a partir del 2010 en adelante, que es una fecha promedio a partir de la cual se acrecienta la tendencia del estudio del cambio ciudadano en los jóvenes.

El análisis de los artículos se realizó en dos momentos: el primero analizar el diseño y metodologías propuestas en los artículos de acuerdo a los años, lo cual se representó en una línea del tiempo.

La segunda parte del análisis fue describir los temas que abordaban y analizar su relación con la comunicación, lo cual se presenta en una tabla que aborda una breve descripción del tema de estudio de cada artículo de acuerdo al año de publicación.

ANÁlisis DE RESULTADOS: ESTADO DEL ARTE

DE LOS ESTUDIOS SOBRE COMUNICACIÓN

\section{Sobre el análisis de la metodología}

Como se ha presentado, los estudios versan en su mayoría sobre la inquietud de comprender el cambio comportamental ciudadano y democrático, en particular en la población joven 
donde se percibe un cambio de actitud. Es relevante que estos estudios buscan incorporar variables que apelan a procesos comunicativos como la actitud frente a los medios, el consumo mediático, el uso de internet, como un elemento sin el cual no se puede explicar el cambio en la percepción y práctica de las generaciones más jóvenes de ciudadanos.

Por esta razón, los estudios, sobre todo en los primeros años, muestran una tendencia tanto exploratoria como descriptiva del fenómeno. Es decir, se busca establecer las bases del comportamiento de los jóvenes y su relación con los medios, esta postura parte del planteamiento de que las prácticas y comprensión de la ciudadanía ha cambiado en los jóvenes, por lo tanto, es necesario explorar y describirla ( $\mathrm{O}^{\prime}$ Donell, 2007). Estos estudios tienen una tendencia mixta, atendiendo a estos dos propósitos, la descripción de comportamientos y la comprensión de los sentidos e incluyen la relación y de los medios digitales como factor relevantes para los jóvenes en sus prácticas ciudadanas (Horbath y Gracia, 20I3; Sola-Morales y Hernández-Santaolalla, 2017; Cano-Correa, Quiroz-Velasco y Nájar-Ortega, 2017; Lozano y Alvarado, 20II).

Se observan algunos estudios de tinte exploratorio (Cogo y Barsi, 20II; Valderrama, 20I3; Avalos, 20I4) quienes se centran en estudiar los sentidos y dándole un papel relevante a lo cualitativo (Ocampo, 20I I). Entre sus metodologías se orientan a los análisis de contenido. Otros estudios tiene una tendencia documental y tipo ensayístico (Cavieres, 2015, Pausch, 20I6).

Además México, Colombia y España han producido varios artículos sobre ciudadanía, jóvenes y comunicación a lo largo de los años, España tiende al diseño cuantitativo y con alcances descriptivos o correlacionales. Países como Colombia, México y Chile muestran una mayor producción de artículos en el tema, aunque con orientaciones metodológicas enfocadas en el trabajo cualitativo y con orientación crítica en las teorías, es decir, exploran las creaciones de sentido a partir de las relaciones estructurales de la sociedad y los espacios comunicativos.

Se presenta a continuación una línea del tiempo que recoge las tendencias metodológicas por año de los artículos revisados del 20II al 20I4 (Figura I).

En estos primeros cuatro años (Figura I) se observa la tendencia exploratoria a través de la dominancia de estudios cualitativos. La razón que presentan los estudios para preferir la metodología de los estudios cualitativos tiene que ver con la necesidad de comprender los sentidos de la ciudadanía, que es parte de lo que se ha cuestionado en las generaciones más jóvenes.

Es cierto que durante estos años existen amplios estudios cuantitativos que tienden a basarse en encuestas nacionales o amplias bases de datos. Sin embargo, estos estudios tienen un enfoque predominantemente político y descriptivo o correlacional y no incorporan el 
análisis de fenómenos o categorías del campo de la comunicación como factores relevantes, salvo los que son parte del trabajo descriptivo, como que medios consumen para informarse de política.

FIGURA 1. ANÁLISIS METODOLÓGICO DE ARTÍCULO ENTRE 2011 Y 2014

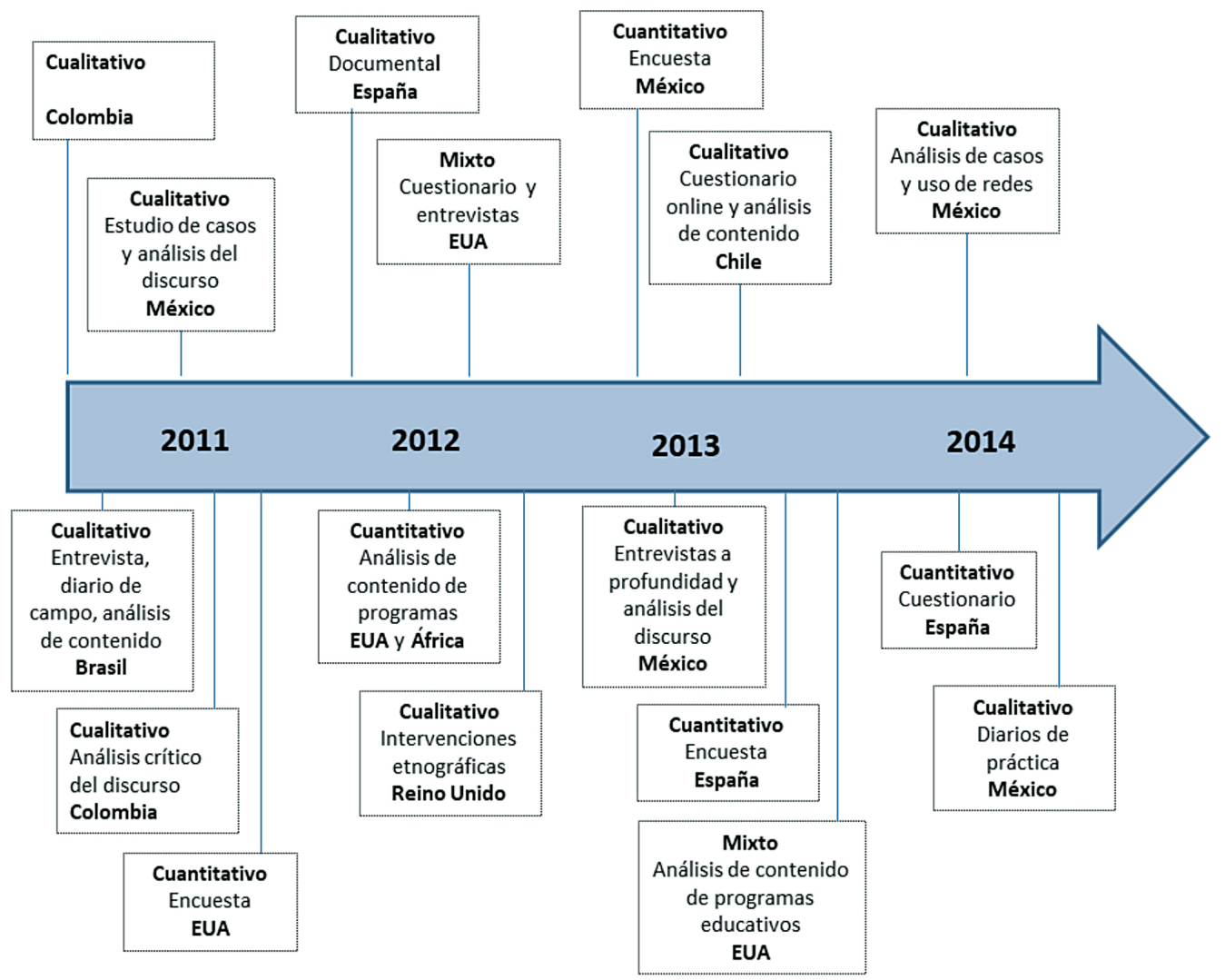

FUENTE: ELABORACIÓN PROPIA.

Con respecto a los años 2015 al 2018 se presenta una segunda parte de la línea del tiempo con las tendencias metodológicas (Figura 2).

En la Figura 2 se observa cómo en el año 2015 y 2016 aún predomina la tendencia a los estudios cualitativos, mientras que a partir del 2017 aumenta la presencia de estudios mixtos. También se hace notar como en este periodo surgen otro tipo de metodologías menos frecuentes y que integran enfoques pedagógicos, como la intervención educativa, el 
análisis legislativo y metodologías más especializadas del campo de la comunicación, como el análisis de contenido en redes y los estudios de audiencia.

FIGURA 2. ANÁLISIS METODOLÓGICO DE ARTÍCULO ENTRE 2015 Y 2018

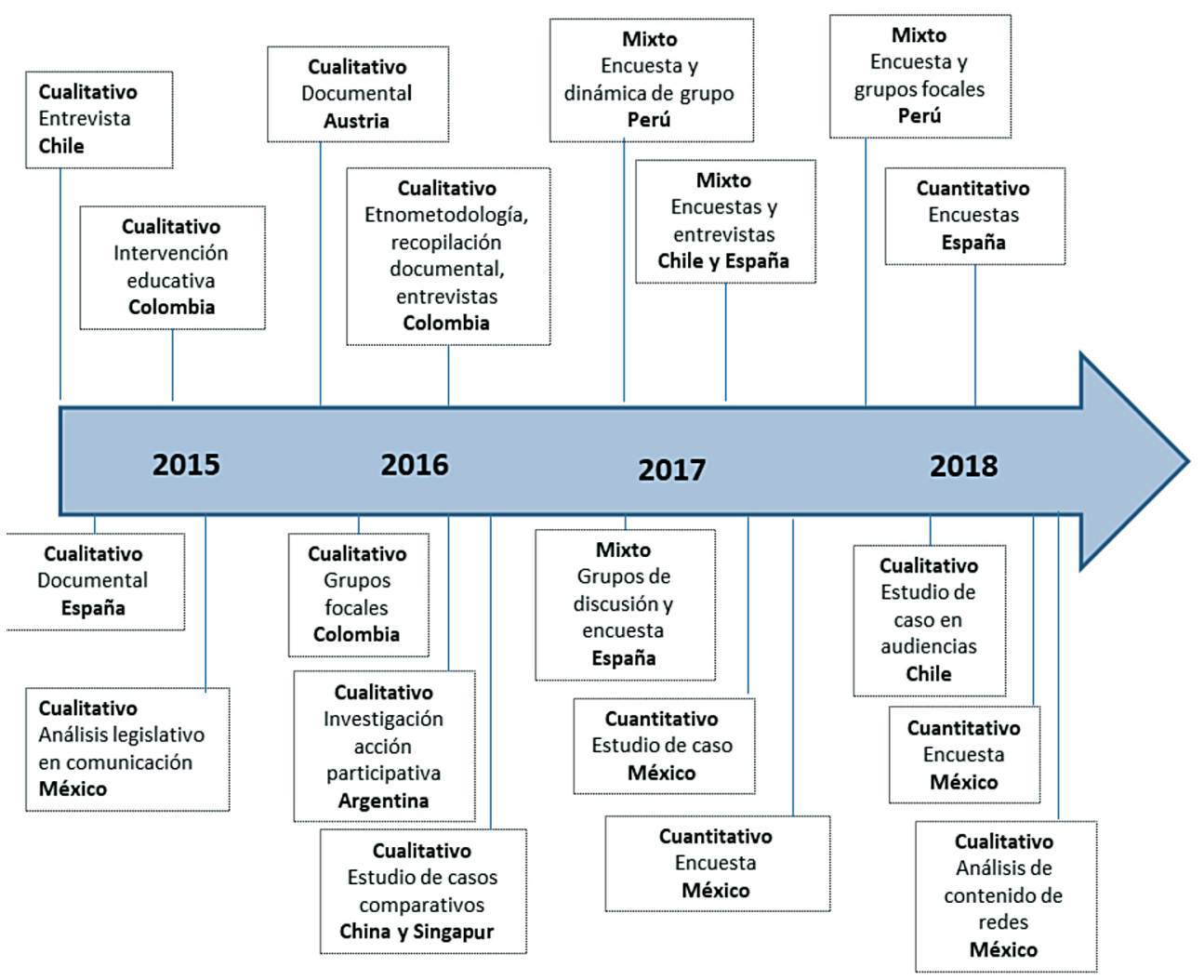

FUENTE: ELABORACIÓN PROPIA.

En estas figuras se observa también cómo la tendencia Latinoamérica es a los estudios cualitativos, mientras que países como Reino Unido, España o Estados Unidos de América tienden a estudios cuantitativos o mixtos (basados en encuestas para la parte cuantitativa).

Como se observa, la tendencia metodológica parece ser la especificidad en el uso de términos, técnicas y objetos de estudio cada vez más definidos dentro del campo de la comunicación. Aunque se observa la permanencia dela orientación hacia los trabajos cualitativos, es cierto que en los últimos años se incrementan los trabajos de tipo mixto. 


\section{SOBRE LA RELACIÓN CIUDADANÍA, JÓVENES Y COMUNICACIÓN}

En cuanto a las categorías que se mencionan, se observa que hay una tendencia de va de realizar estudios generales que hablan de comunicación o interacción como categoría general, pero que no incluyen aspectos referenciales de las teorías o modelos de comunicación, y el uso de términos se muestra desdibujado, a una tendencia que aborda fenómenos específicos de la comunicación en contextos específicos y con categorías comunicativas.

En los primeros estudios analizados se hace mención a categorías comunicativas, pero no se desarrollan o contemplan con claridad suficiente en los estudios, y hay una carencia de marcos referenciales de las teorías de comunicación para la comprensión de los fenómenos.

Sin embargo, en los últimos años se observa que los estudios que abordan contextos específicos (como las organizaciones, ciudades o movimientos) aumentan, así como los contextos de interacción específica (relación con medios y competencias mediáticas, usos digitales para causas ciudadanas y relación de discursos gubernamentales de formación ciudadana con prácticas).

En los siguientes cuadros ( I y 2) se presentan los temas que abordan los estudios y el país al que corresponden.

En el año 20II, se observa la incorporación de conceptos como las percepciones, los usos de medios, las apropiaciones y los discursos. Aunque estos estudios, como se ha mencionado, tienen una tendencia exploratoria o descriptiva sobre la ciudadanía en los jóvenes, buscan establecer una relación con las prácticas ciudadanas actuales (Lozano y Alvarado, 20II; Salzman y Albarran, 20 Ir; Cogo y Barsi, 20II; Morfín, 20II; Ocampo, 20II).

En el año 20I2, se hace notar la presencia de los estudios de alfabetización mediática ciudadana, estos estudios tienen una visión pedagógica que es la de la formación del pensamiento crítico de la audiencia, así como la formación ciudadana en los programas educativos en los medios de comunicación. Entre las visiones que integran el enfoque pedagógico con fuerte énfasis a alfabetización mediática y relación con los medios de comunicación, son los países como EUA, España y Reino Unido. Estudios como el realizado en EUA y África que proponen una alfabetización centrado tanto en las competencias mediáticas como en el impacto en el compromiso cívico (Culver y Jacobson, 20I2), o el estudio de Reino Unido que se centran en las interacciones culturales mediáticas (Kendall y McDougall, 20I2). Otros estudios se centran en la alfabetización y la interacción con otros espacios como los urbanos (Carmen y Aguaded, 20I2; Obar, Zube y Lampe, 2012).

En el año 2013, se presentan estudios que tienen relación con contextos específicos, por ejemplo a las protestas en los medios digitales o en organizaciones. Estos estudios dan mayor papel al estudio de las interacciones (Valderrama, 20I3; Izquieta y Callejo, 20I3; 
Middaugh y Kahne, 2013). Algunos otros se enfocan en la influencia cultural (Horbath y Gracia, 2013; Mata-Benito, Ballesteros y Padilla, 2013).

En el año 20I4, se ve con mayor claridad la tendencia de ligar la ciudadanía juvenil con el ambiente digital, además se muestra una mayor relevancia en el papel de las experiencias y habilidades del sujeto-receptor o audiencias (Padilla de la Torre, 20I4; García- Ruiz, Ramírez-García y Rodríguez-Rosell, 20I4).

Con respecto a los años 2015 al 20I8, se presenta el Cuadro 2.

\section{CUADRO 1. AGRUPACIÓN DE TEMAS QUE ABORDAN LOS ARTÍCULOS}

Y SUS PAÍSES DE ORIGEN DEL 2011 AL 2014

\begin{tabular}{|l|l|l|l|}
\multicolumn{2}{|c|}{2011} & \multicolumn{2}{c|}{2013} \\
$\begin{array}{l}\text { Percepción de } \\
\text { ciudadanía y detonantes } \\
\text { para prácticas políticas } \\
\text { Colombia }\end{array}$ & $\begin{array}{l}\text { Alfabetización mediática } \\
\text { ciudadana } \\
\text { España }\end{array}$ & $\begin{array}{l}\text { Cultura política, } \\
\text { prácticas ciudadanas y } \\
\text { uso de medios } \\
\text { México }\end{array}$ & $\begin{array}{l}\text { Prácticas políticas en } \\
\text { Internet } \\
\text { México }\end{array}$ \\
\hline $\begin{array}{l}\text { Uso de Internet } \\
\text { y características } \\
\text { de los jóvenes } \\
\text { latinoamericanos }\end{array}$ & $\begin{array}{l}\text { Activismo ciudadano y } \\
\text { uso de redes sociales } \\
\text { EUA }\end{array}$ & $\begin{array}{l}\text { Apropiación de } \\
\text { tecnologías para } \\
\text { acciones de protestas } \\
\text { Chile }\end{array}$ & $\begin{array}{l}\text { Experiencias juveniles de } \\
\text { protestas y uso de redes } \\
\text { México }\end{array}$ \\
\hline $\begin{array}{l}\text { Apropiación y uso de } \\
\text { tecnologías en jóvenes } \\
\text { en ONG } \\
\text { Brasil }\end{array}$ & $\begin{array}{l}\text { Alfabetización mediática } \\
\text { yparticipación } \\
\text { ciudadana } \\
\text { EUA y África }\end{array}$ & $\begin{array}{l}\text { Estudio del discurso } \\
\text { ciudadano y } \\
\text { significación } \\
\text { México }\end{array}$ & \\
\hline $\begin{array}{l}\text { Participación política, } \\
\text { discurso y acciones } \\
\text { colectivas } \\
\text { México }\end{array}$ & $\begin{array}{l}\text { Cambios en } \\
\text { participación } \\
\text { ciudadana y su } \\
\text { relación con las ong } \\
\text { España }\end{array}$ & $\begin{array}{l}\text { Competencia mediática } \\
\text { y relación con prácticas } \\
\text { ciudadanas } \\
\text { España }\end{array}$ \\
$\begin{array}{l}\text { Discurso sobre } \\
\text { ciudadanía y relación } \\
\text { con juventud y el Estado } \\
\text { Colombia }\end{array}$ & $\begin{array}{l}\text { Interacción con medios } \\
\text { digitales y compromiso } \\
\text { ciudadano } \\
\text { EUA }\end{array}$ & \\
\hline
\end{tabular}

FUENTE: ELABORACIÓN PROPIA. 
CUADRO 2. AGRUPACIÓN DE TEMAS QUE ABORDAN LOS ARTÍCULOS

Y SUS PAÍSES DE ORIGEN DEL 2015 AL 2018

\begin{tabular}{|c|c|c|c|}
\hline 2015 & 2016 & 2017 & 2018 \\
\hline $\begin{array}{l}\text { Enseñanza de la } \\
\text { ciudadanía en el conflicto } \\
\text { Chile }\end{array}$ & $\begin{array}{l}\text { Significación y educación } \\
\text { ciudadana } \\
\text { Austria }\end{array}$ & $\begin{array}{l}\text { Percepción ciudadana } \\
\text { y relación con medios } \\
\text { de comunicación } \\
\text { Perú }\end{array}$ & $\begin{array}{l}\text { Percepción y discurso } \\
\text { ciudadano } \\
\text { México }\end{array}$ \\
\hline $\begin{array}{l}\text { Formación, interacción y } \\
\text { participación ciudadana } \\
\text { en instituciones } \\
\text { educativas } \\
\text { Colombia }\end{array}$ & $\begin{array}{l}\text { Concepto de ciudadanía y } \\
\text { comunicación comunitaria } \\
\text { Colombia }\end{array}$ & $\begin{array}{l}\text { Abstención política } \\
\text { y nuevas formas de } \\
\text { participación } \\
\text { Chile y España }\end{array}$ & $\begin{array}{l}\text { Conductas de } \\
\text { ciudadanía digital } \\
\text { España }\end{array}$ \\
\hline $\begin{array}{l}\text { Experiencia cotidiana } \\
\text { de la ciudad como } \\
\text { constructora de } \\
\text { ciudadanía } \\
\text { España }\end{array}$ & $\begin{array}{l}\text { Marcos de acción } \\
\text { organizacional, interacción } \\
\text { y jóvenes } \\
\text { Colombia }\end{array}$ & $\begin{array}{l}\text { Usos y prácticas } \\
\text { ciudadanas online y } \\
\text { offline de jóvenes } \\
\text { España }\end{array}$ & $\begin{array}{l}\text { Capacidad crítica } \\
\text { de jóvenes frente } \\
\text { a noticias de } \\
\text { movimientos sociales } \\
\text { Chile }\end{array}$ \\
\hline \multirow{2}{*}{$\begin{array}{l}\text { Análisis de cambios } \\
\text { legislativos en materia } \\
\text { de telecomunicaciones } \\
\text { México }\end{array}$} & $\begin{array}{l}\text { Educación ciudadana, } \\
\text { interacción y } \\
\text { organizaciones sociales } \\
\text { Argentina }\end{array}$ & $\begin{array}{l}\text { Formación de } \\
\text { ciudadanía en } \\
\text { organizaciones } \\
\text { México }\end{array}$ & $\begin{array}{l}\text { Indicadores de } \\
\text { ciudadanía digital } \\
\text { México }\end{array}$ \\
\hline & $\begin{array}{l}\text { Discursos de educación } \\
\text { cívica en ciudades, prácticas } \\
\text { ciudadanas, contexto y } \\
\text { significado } \\
\text { China y Singapur }\end{array}$ & $\begin{array}{l}\text { Efectos de los medios } \\
\text { y campañas cívicas } \\
\text { México }\end{array}$ & $\begin{array}{l}\text { Participación en Twitter } \\
\text { de movilizaciones } \\
\text { sociales } \\
\text { México }\end{array}$ \\
\hline
\end{tabular}

FUENTE: ELABORACIÓN PROPIA.

En el año 2015, continúa la línea de artículos que dan importancia al aspecto pedagógico, es decir, resaltan la necesidad de formar en ciudadanía y estudiar ese proceso a partir de las interacciones, apropiaciones y respuestas. También en este año se observa la introducción de aspectos estructurales que analizan los factores estructurales asociados al conflicto (Cavieres, 2015), el papel de las instituciones educativas (Ceballos, 2015), los aspectos urbanos 
y el poder público (Benedicto, 20I6), los que abordan las medidas legislativas que afectan la construcción de la imagen de los medios de comunicación (Ortega, 2015).

En el 20I6, continúa la tendencia de incorporar elementos estructurales de la sociedady la ciudadanía, así como la presencia de la conciencia de la comunicación comunitaria (Huertas y Arboledas, 20I6; Uranga, 20I7), el papel de las instituciones democráticas (Pausch, 2016), el papel de los discursos de formación ciudadana en las grandes estructuras urbanas (Alviar-Martín y Baildon, 20I6) y los que estudian la cultura organizacional y los vínculos y narrativas internas (Ortiz-Ruiz, 2016).

En el 2017, los estudios incorporan la relación con los medios de comunicación, en particular las redes sociodigitales. Algunos se centran en la influencia de los medios en la percepción de la ciudadanía (Cano-Correa, Quiroz-Velasco y Nájar-Ortega, 20I7), comparación de usos online y offline (García, Fernández y Porto, 20I7), vínculo de la organización y sus mensajes con perfiles ciudadanos (Hernándezy Alcántara, 2017), efectos de los medios, apropiación y comportamiento (Díaz Jiménez, 2017), y nuevas formas de participación (Sola-Morales y Hernández-Santaolalla, 2017).

Para el 20I8, observamos que la tendencia de los artículos es el estudio de la ciudadanía y comunicación en contextos o medios específicos, como en las universidades (Quiroz, 20I8), en el ambiente digital (Lozano y Fernández-Prados, 20I8), en las redes sociodigitales y la relación como audiencia ante movimientos sociales (Vernier, Montero y Schieling, 20I8; Giraldo-Luque, Fernández-García y Pérez-Arce, 20I8), lo digital y las universidades (Martínez y Ramírez, 20I8).

\section{DISCUSIONES}

La ciudadanía es estudiada desde los abordajes políticos y de cultura democrática, de lo cual es posible encontrar una gran variedad de artículos aunque incluyen en sus conclusiones la referencia a la necesidad de estudiar las interacciones y aspectos comunicativos. Sin embargo, cuando se incluye la variable de la comunicación dentro de la investigación, la producción se reduce significativamente o se limita a campos específicos como los estudios organizacionales, estudios de los medios o de alfabetización mediática.

Los estudios de ciudadanía han pasado de estudiar la ciudadanía como un fenómeno general a estudiarlo en actos o movimientos concretos, lo cual muestra una mayor definición de la comprensión de la ciudadanía en los jóvenes como de una claridad mayor de los aspectos relevantes de la configuración ciudadana. Los movimientos sociales en particular son de gran interés para los estudios de la ciudadanía, y es en estos estudios donde se aborda la 
relevancia de los medios de comunicación y en especial las redes digitales como los espacios de construcción política de las nuevas generaciones.

Existe una tendencia innegable de asociación de la ciudadanía y lo digital cuando se habla de los jóvenes, por lo que es una de las categorías o variables más usadas en los estudios. El estudio de lo digital y los jóvenes se ha convertido en un binomio inseparable para comprender la ciudadanía.

Hay algunas tendencias a incorporar análisis micro y macro de la comunicación para la construcción ciudadana, aunque los esfuerzos quedan poco claros en cuestiones metodológicas, resulta de interés el énfasis de varios artículos de analizar la ciudadanía en conjunto con la comunicación como un fenómeno que siempre debe abordar lo estructural como las significaciones propias y características del sujeto.

\section{CONCLUSIONES}

Con respecto a los estudios sobre ciudadanía en la juventud y aspectos de comunicación, pocas veces se aborda desde las teorías de la comunicación, aunque sí se incorporan categorías de análisis del campo.

Los estudios de ciudadanía que se abordan desde la comunicación van en aumento, con la tendencia al estudio de movimientos, contextos o grupos específicos así como de sus interacciones.

Se infiere que el cambio en la aproximación teórico-conceptual proviene de la influencia de las corrientes desde las cuales se aborda el tema de la ciudadanía: la influencia pedagógica y el incremento del discurso sobre educación para la ciudadanía ha influido los estudios.

Con respecto al análisis por regiones, se observa que en Latinoamérica los estudios conservan una corriente que se focaliza en el estudio de los movimientos sociales, conserva la línea tradicional de estudios de postura crítica hacia el sistema y donde los medios de comunicación se estudian con una mirada negativa como reproductores de desigualdades. Mientras que otros países como Reino unido, Estados Unidos o Austria muestran una mirada que ha asumido la presencia de los medios como parte de la cultura y no como algo en contra de ella, de tal forma que la línea de los estudios se centra más en la alfabetización mediática, el estudio de las audiencias y su educación.

Es posible que el crecimiento tanto de la exposición como del acceso a la tecnología, y la influencia del mundo virtual en la vida de los jóvenes ha marcado una fuerte tendencia a estudiar la virtualidad, sus interacciones y medios como un espacio propio de la ciudadanía juvenil. Esto se ha reforzado por la apropiación del Internet como herramienta que fortalece 
los movimientos sociales, de esta forma se infiere que la participación virtual ha unido el estudio de la comunicación con el estudio de la ciudadanía.

En cuanto a aportaciones a la metodología, se observa una tendencia marcada a los estudios cualitativos sobre todo en Latinoamérica, aunque en los últimos años hay un incremento de estudios mixtos. En cuanto a técnicas de investigación, se observan ciertas aportaciones como las orientadas a intervenciones educativas e investigación-acción, además de la presencia cada vez mayor de metodologías propias del estudio de la comunicación como el análisis de consumo mediático, de recepción y audiencias.

Con respecto a este cambio de rumbo metodológico, la tendencia creciente de incorporar estudios mixtos se supone como una respuesta ante la insuficiencia de los estudios cuantitativos para explicar el porqué del cambio en las prácticas y sentidos ciudadanos en los jóvenes, y que buscaban describir el comportamiento ciudadano a partir de variables sociodemográficas. Esta necesidad de explicar un cambio tan fundamental para la democracia, como es la participación en una nueva generación de ciudadanos, se deduce como un factor que ha generado apertura para incorporar elementos comunicativos como la interacción, la creación de sentidos, la mediación y la apropiación, y que amplían la posibilidad de explicar los fenómenos sociales.

El estudio de la ciudadanía de ser abordado como un estatus jurídico, ahora se estudia cada vez más como una interacción comunicativa capaz de crear sentidos, no solo de reproducirlos. De ahí la creciente necesidad de una mirada multidisciplinaria que incorpore teorías comunicativas y psicológicas para su comprensión.

Sin embargo, tampoco se observa que dentro de los estudios de comunicación haya una línea marcada del estudio de la ciudadanía, ni dentro de los estudios de ciudadanía se observa una incorporación conceptual y estructural de teorías de la comunicación. Esto puede deberse a que las teorías de la comunicación y la comunicación como ciencia son un esquema en desarrollo que no tiene aún un espacio claro dentro del estudio de las ciencias sociales, y que por tanto existe un desconocimiento de las posibilidades de la comunicación para brindar marcos referenciales y no solo orientaciones técnicas. 


\section{REFERENCIAS}

Alviar-Martin, T. y Baildon, M.C. (20I6). Context and curriculum in two global cities: a study of discourses of citizenship in Hong kong and Singapore. Education policy analysis archives, 24(58). http://dx.doi.org/10.14507/epaa.24.2140

Avalos, J.M. (20I4). Disidencias juveniles y medios digitales en México: ¿una coyuntura con elementos de futuro para la participación política? Argumentos, 27(75), 47-I70.

Benedicto, J. (20I6). La ciudadanía juvenil: un enfoque basado en las experiencias vitales de los jóvenes. Revista Latinoamericana de Ciencias Sociales, Niñez y Fuventud, I4(2), 925-938.

Cano-Correa, A., Quiroz-Velasco, M. y Najar-Ortega, R. (2017). Jóvenes universitarios en Lima: política, medios y participación. Revista Científica de Educomunicación, 53(25), 71-79.

Carmen, M. y Aguaded, J.I. (20I2). La ciudad, espacio de educomunicación y participación de la ciudadanía alfabetizada. Revista Latinoamericana de Ciencias de la Comunicación, 9(16), 54-63.

Cavieres, E. (2015). Enseñando ciudadanía en medio del conflicto. Revista Mexicana de Investigación Educativa, 20(67), I3II-I334.

Ceballos, J. (2015). Medios de comunicación escolar, educación y ciudadanía. Una mirada desde las mediaciones (Tesis doctoral). Argentina, Universidad Nacional de La Plata. Recuperado de http://sedici.unlp.edu.ar/handle/Iog15/47712

Cogo, D. y Barsi, D. (20II). Movimientos juveniles, medios digitales y prácticas de ciudadanía en Fortaleza (Brasil). Observatorio fournal OBS, 5(I), 035-057.

Culver, S.H. y Jacobson, T. (2OI2). Alfabetización mediática como método para fomentar la participación cívica. Comunicar, (39) (XX), 73-80.

Díaz, O. (20I7). Las campañas electorales, los medios y su impacto en el compromiso cívico de los mexicanos en la elección presidencial de 2012. Comunicación y Sociedad, (29), I39-164.

García, M., Fernández, C. y Porto, L. (20I7). Empoderamiento de los jóvenes a través de las redes sociales. Construcción de una ciudadanía digital comprometida. Comunication \&o Society,30(3), I29-I4O.

García-Ruiz, R., Ramírez-García, A. y Rodríguez-Rosell, M. (20I4). Media literacy education for a new prosumer citizenship. Comunicar, (43) (XXII), I5-23.

Giraldo-Luque, S. y Fernandez, N. y Pérez-Arce, J. (20I8). La centralidad temática de la movilización \#NiUnaMenos en Twitter. El Profesional de la Información, (27), 96.

Hernández, M.C. y Alcántara, N. (2017). Construcción de ciudadanía en organizaciones sociales: propuesta de un marco analítico. Sociológica, (92), 99-139.

Horbath, J. E., y Gracia, M. A. (20I3). La participación ciudadana de los jóvenes mexicanos en la construcción social y democrática del país al inicio del siglo XXI. Revista de Relaciones Internacionales, Estrategia y Seguridad, 8(I),I37-I60. Recuperado de https://www.redalyc.org/articulo. oa?id=927/92729194007

Huertas, O. y Arboledas, A. (2016). Educación ciudadana en el marco del pensamiento complejo. Revista lasallista de investigación, I3(2), I28-135. 
Kendall, A. y McDougall, J. (20I2). Alfabetización mediática crítica en la postmodernidad. Comunicar, (38) (XIX), 2 I-29.

Izquieta, J.L. y Callejo, J.J. (20I3). Asociacionismo y participación voluntaria de los jóvenes españoles. Cambios y tendencias actuales. Cuadernos de trabajo social, 26(I), I59-I70.

Livingstone, S. (2008). Taking risky opportunities in youthful content creation: Teenagers' use of social networking sites for intimacy, privacy and self-expression. New Media \&o Society, IO(3), 393-4II.

Lizcano, F. (2012). Conceptos de ciudadano, ciudadano y civismo. Polis, (32). Recuperado de http://journals.openedition.org/polis/6581

Lozano, M. C. y Alvarado, S. V. (2OII). Juicios, discursos y acción política en grupos de jóvenes estudiantes universitarios de Bogotá. Revista Latinoamericana de Ciencias Sociales, Niñez y Zuventud, $I(9)$, IOI-II3.

Lozano-Díaz A. y Fernández-Prados, J. (20I8). Ciudadanía digital y su medida: propiedades psicométricas de una escala y retos para la educación superior. Education in the Knowledge Society (EKS), I9(3), 83-Ior. Recuperado de https://revistas.usal.es/index.php/eks/article/view/18335

Martínez, K. y Ramírez, A. (20I8). Ciudadanía digital para practicar un gobierno abierto: análisis del uso de las Tecnologías de la Información y de la Comunicación en un ambiente universitario. Revista Especializada en Investigación Furídica.

Mata-Benito, P., Ballesteros, B. y Padilla-Carmona, M. (2013). Ciudadanía participativa y transformadora: análisis de discursos y propuestas de aprendizaje. Teoría de la Educación. Revista Interuniversitaria, (25), 49-68.

Meyer, D. (200I). Disaffected Democracies: What's Troubling the Trilateral Countries, edited by Susan Pharr and Robert Putnam, Princeton, NJ: Princeton University Press (2000). Reviewed by David S. Meyer. Fournal of Political Ecology, (8), 84.

Middaugh, E. y Kahne, J. (20I3). Nuevos medios como herramienta para el aprendizaje cívico. Comunicar, (40) (XX), 99-108.

Morfín, C. (20II). Jóvenes en acciones colectivas y movimientos sociales para redefinir los espacios públicos y las prácticas ciudadanas. Revista Latinoamericana de Ciencias Sociales, Niñez y Fuventud, I(9), 6I-79.

Obar, J.A., Zube, P. y Lampe, C. (2012). Advocacy 2.0: an analysis of how advocacy groups in the United States perceive and use social media as tools for facilitating civic engagement and collective action. Penn State Univesity Press. Fournal of information policy, 2, I-25.

Ocampo, A. M. (20II). Ciudadanía juvenil y estado: discursos de gobierno sobre sus significados. Revista Latinoamericana de Ciencias Sociales, Niñez y Fuventud, I(9), 287-303.

O’Donell, G. (2007). Las crisis perpetuas de la democracia. Revista Polis, 3(I), II-20.

Ortega, P. (2015). Participación ciudadana y medios públicos. Revista Latinoamericana de Ciencias de la Comunicación, I2(23), 193-203.

Ortiz-Ruiz, N. (2016). ¿Qué mueve a las organizaciones juveniles? Revista Latinoamericana de Ciencias Sociales, Niñez y Fuventud, I4(I), 53 I- 543. 
Padilla de la Torre, M. R. (20I4). Ciudadanía política en la red. Análisis de las prácticas políticas entre jóvenes universitarios. Comunicación y Sociedad, (2I), 7I-IOO. Recuperado de https://www.redalyc.org/articulo.oa?id=346/34631113004

Pausch, M. (2016). Citizenship education in time of crisis. Foro de Educacion, I (20), 3-9.

Quiroz, T. (20I8). Universitarios, ciudadanía y competencias mediáticas. Revista Lumina, I2(I), 6-16.

Salzman, R. y Albarran, A. (20II). Internet use in Latin America. Palabra Clave, I4(2). 297-3I3.

Sola-Morales, S. y Hernández-Santaolalla, V. (20I7). Abstención política y nuevas formas de participación política de los jóvenes: análisis comparativo entre Chile y España. Revista Latina de Comunicación Social, (72), 629-648.

Uranga, W. (2017). Comunicar para la incidencia política. Revista Latinoamericana de Ciencias de la Comunicación, I3(25).

Valderrama, L.B. (20I3). Jóvenes, ciudadanía y tecnologías de información y comunicación. El movimiento estudiantil chileno. Revista Latinoamericana de ciencias sociales, niñez y juventud, II(I), I23-235.

Vernier, M., Cárcamo, L.y Scheiling, E. (2018). Pensamiento crítico de los jóvenes ciudadanos frente a las noticias en Chile. Comunicar: Revista científica iberoamericana de comunicación y educación, (54), IoI-IIo. https://doi.org/IO.3916/c54-20I8-IO 